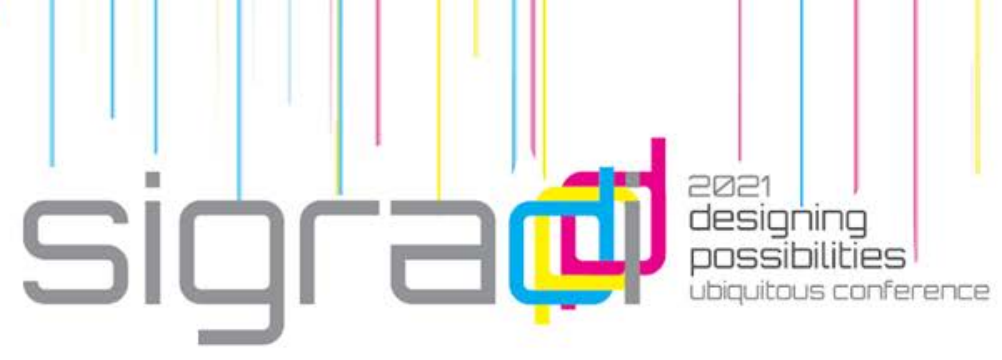

\title{
Visualizing Connections: Social Infrastructure at Two Brazilian Universities
}

\author{
Luís Henrique Pavan ${ }^{1}$, Camila Poeta Mangrich ${ }^{1}$, Lucas Fernandes de \\ Oliveira $^{1}$, Gabriela Peglow Harthmann ${ }^{1}$, José Ripper Kós ${ }^{1}$ \\ ${ }^{1}$ Universidade Federal de Santa Catarina, Brasil \\ luis.henrique.pavan@posgrad.ufsc.br \\ camila.poeta@ufsc.br \\ fernandes.lucas@grad.ufsc.br \\ gabriela.harthmann@grad.ufsc.br \\ jose.kos@ufsc.br
}

\begin{abstract}
Although often underrecognized, social infrastructures are a crucial ordering element in cities, as they are places or associations that shape the way people interact. We studied The Federal University of Rio de Janeiro (UFRJ) and the Federal University of Santa Catarina (UFSC) in three different diagrammatic approaches. An urban approach considered the relation between the institutions and their host cities, emphasizing the social infrastructure provision. A second analysis considered institutional activities, emphasizing how typological arrangements in which they occur. The third study focused on the UFSC campus and framed its social infrastructure through a systemic approach, focusing on the interactions between distinct community services and their distribution on campus. In addition to the diagrammatic emphasis allowing the interactions between the university and society to be clearly visualized, the results highlight a systemic disposition that reinforces the university as an urban entity and a crucial municipal and regional social support.
\end{abstract}

Keywords: Social infrastructure, Data visualization, University campus, Complex systems, Public universities

\section{Introdução}

Os campi universitários têm sido objeto de frequente interesse científico nas últimas décadas (Taylor, 2016). As universidades amparam boa parte do desenvolvimento educacional, tecnológico e econômico das nações sendo o campus a manifestação em território das organizações acadêmicas enquanto 
instituição. A pandemia da COVID-19 refletiu-se brutalmente na educação, obrigando a interrupção de atividades presenciais, a adaptação curricular momentânea ao modelo remoto e ainda a própria postura de assistência social estudantil que as universidades prestam. Pode-se afirmar que a crise sanitária tornou crucial o papel de infraestrutura social que essas instituições desempenham, amparando parte vital e muitas vezes não reconhecida da sociedade e apresentando aspectos fundamentais das dinâmicas de sociabilidade humanas.

As infraestruturas têm ganhado relevância científica à medida em que publicações abordam teorias gerais de infraestrutura colocando em primeiro plano, para além da facilitação, seus aspectos geralmente ocultos, sejam materiais, informacionais ou ideológicos. Mesmo há mais tempo, escritos como Winner (1980) traziam à luz alguns aspectos pouco evidentes da construção infraestrutural, legando a eles aspectos políticos indissociáveis. À medida que ordenam a vida na cidade, estes dispositivos possibilitam diferentes arranjos, cada qual com implicações sociais inerentes. As sociedades optam por tecnologias com impacto direto no comportamento das pessoas, alterando como trabalham, consomem, deslocam-se e socializam. A esse último, faz-se a ressalva que deve ser sempre considerada sua conectividade com os outros aspectos infraestruturais, ponto no qual apoia-se o conceito de infraestrutura social aqui explorado.

Principalmente porque esses aspectos são pouco visíveis isoladamente, este trabalho objetiva explorar diagramaticamente as correlações das infraestruturas sociais e as tipologias do campus universitário em duas universidades públicas brasileiras. Essas infraestruturas sociais são localidades e organizações nem sempre vinculados a departamentos ou administradas de forma conjunta, uma característica não necessariamente negativa, mas que dificulta a visão sistêmica das universidades. Como objetos de estudo foram escolhidos os principais campi da Universidade Federal de Rio de Janeiro (UFRJ) e da Universidade Federal de Santa Catarina (UFSC). O campus daquela, chamado de Cidade Universitária, localizado na Ilha do Fundão e o desta localizado no bairro Trindade, em Florianópolis.

\section{Revisão de Literatura}

O conceito de infraestrutura social é relativamente recente e teve maior alcance a partir das constatações de Eric Klinenberg (2018). Partindo da onda de calor que atingiu Chicago em 1995, o sociólogo relata uma sequência de 
eventos que colapsaram o sistema infraestrutural da cidade. Além da alta demanda de energia que provocou falhas no bombeamento hidráulico de inúmeros locais, os reflexos do evento climático foram piores onde a segregação social e a vulnerabilidade econômica eram acentuadas, levando centenas de pessoas à óbito. Porém, ao investigar com atenção determinados locais, Klinenberg observou maior resiliência à crise em grupos nos quais predominavam vínculos sociais, mesmo diante de limitações econômicas. A diferença determinante nesses locais demograficamente semelhantes estava no que ele definiu como infraestrutura social, ou seja, nos locais físicos e organizações que moldam a maneira pela qual as pessoas interagem.

Latham e Layton (2019), após expressiva revisão de literatura sobre as infraestruturas sociais, acrescenta interpretações e novas associações acerca da teoria geral de infraestruturas. Vendo-as como facilitadores de atividades, os autores correlacionam as qualidades materiais, o design e a distribuição das infraestruturas sociais às outras redes de serviços e como provisionam diferentes usos, afetando decisivamente seu funcionamento no sistema. Eles atribuem ainda aos cientistas sociais o papel de dar sentido e articular as maneiras pelas quais essas infraestruturas sociais podem ser praticadas e porque elas têm importância, sobretudo porque são raramente percebidas.

Estudando planos diretores de 50 universidades norte-americanas, Hajrasouliha (2017) estabeleceu sete dimensões formais dos territórios universitários. As dimensões por ele encontradas consistem em organização do uso da terra; densidade; conectividade; configuração espacial; habitação do campus; espaços verdes e contexto. Avalia-se ainda como essa disposição espacial pode abranger a missão e os objetivos das universidades. Assim, para cada dimensão formal apresentam-se ações correspondentes ao desenho. Apesar de centrada nos Estados Unidos, podemos traçar significativos paralelos entre a formulação territorial dos campi norte-americanos e dos brasileiros. Nesse sentido, os apontamentos de Hajrasouliha constituem ferramentas de análise úteis, apreciadas as diferenças de contextos. Serão mais atentamente analisadas aqui a dimensão contextual e da conectividade. $\mathrm{Na}$ contextual identifica-se uma dependência da administração universitária como ponto decisivo, principalmente nas interações comunitárias urbanas e com entes privados, sendo crucial a adequação às características socioeconômicas da comunidade circundante. Nessa relação contextual, o projeto implica diretamente promover ou enfraquecer as interações entre o campus e seu contexto. As principais ações encontradas pelo autor vão no sentido da construção de aliança com as cidades e oportunizar o campus como destino público. O grau de integração da rede viária do campus e da área circundante é avaliado na dimensão da conectividade. As ações de desenho incluem a provisão de entradas atrativas e rotas peatonais claras, aproximando 
fisicamente as principais atividades e destinos e estabelecendo fortes conexões entre os bairros vizinhos e o campus.

A história das universidades confunde-se com as discussões sobre a relação entre campus e cidade, de escolas isoladas a conjuntos edificados de grande porte. Pinto e Buffa (2009) apontam as conexões existentes entre as políticas educacionais, os modelos de ensino superior, a arquitetura e o urbanismo de alguns campi brasileiros. A materialização dos campi eram desafios não só pelos dispendiosos recursos envolvidos, mas pela originalidade do problema que se colocava aos profissionais e pelo ineditismo do programa proposto. Com cursos atrelados ao atendimento a comunidade, os campi das universidades federais brasileiras tem se reafirmado enquanto importante congregador de sociabilidades e de serviços desde a sua fundação.

A UFRJ, fundada em 1920, e a implantação de seu principal campus na cidade do Rio de Janeiro têm longas histórias. Em 1934, Gustavo Capanema dá início às discussões para definir a localização da primeira universidade pública brasileira, posteriormente chamada de Universidade do Brasil. Outras propostas de inserção urbana do campus foram apresentadas por nomes como Le Corbusier e Lúcio Costa. Esses estudos foram desconsiderados e a decisão pela ilha artificial para abrigar a Cidade Universitária (nome oficial do campus) deu-se em 1948. O plano elaborado tinha ênfase no zoneamento, setorizando distintamente a administração, as unidades acadêmicas, alojamentos e serviços auxiliares (Alberto, 2003). Além da Cidade Universitária, a universidade se desenvolveu em outras localidades, com campus em Macaé, na Praia Vermelha, em Duque de Caxias, bem como unidades isoladas, a exemplo do Centro de Arte Hélio Oiticica, da Escola de Música, da Maternidade Escola e do Museu Nacional.

As discussões para definir a localização da UFSC em Florianópolis partem de 1950, dez anos antes da sua fundação pelo governo federal. A implantação do campus no bairro da Trindade foi aprovada diante muita resistência administrativa e estudantil, dada sua distância das infraestruturas de moradia e transporte, à época concentradas no centro da cidade (Neckel \& Küchler, 2010). O primeiro plano diretor foi concebido entre $1955-56$ pela equipe de Hélio de Queiroz Duarte e Ernest Robert de Carvalho Mange e, apesar de não ter sido integralmente construído, estabeleceu grande parte do traçado viário do campus. Em 1970, encomendou-se um projeto de urbanização e paisagismo a Roberto Burle Marx com execução limitada à Praça da Cidadania. Apesar do seu aspecto de centralidade, diferente da UFRJ, a principal estrutura da UFSC desenvolveu-se de maneira segregada em relação à cidade, fragilizando seus aspectos cívicos e sociais (Kós et al., 2020). 


\section{Metodologia e Resultados}

Considerando o território da UFRJ e da UFSC, o procedimento parte de uma avaliação qualitativa das correlações entre as infraestruturas sociais e suas derivações informais. Os diagramas foram desenvolvidos em ferramentas gráficas de código aberto, explorando três potencialidades de visualização das informações levantadas na curadoria online de plataformas abertas.

\subsection{Estudo 1}

A primeira etapa consistiu em uma aplicação crítica do estudo diagramático desenvolvido em Hajrasouliha (2017) para análise dos campi norte-americanos, consideradas aqui apenas as dimensões contexto e conectividade, dispostas no centro da Figura 1. Adaptando ao contexto brasileiro, foram analisadas as estruturas da UFRJ e da UFSC em suas respectivas cidades-sede. Ambas análises utilizaram bases cartográficas do OpenStreetMaps (OSM) manuseadas na ferramenta QGIS e, exclusivamente na segunda, a plataforma online Kepler.gl foi utilizada para visualização dos dados.

A primeira análise apresentada na Figura 1 focaliza o principal campus de cada uma das instituições, expandidas aos demais campi e unidades distribuídas nos contextos urbanos mais centrais. Nota-se que, embora tendo seu principal campus isolado em uma ilha, a UFRJ (A) dispõe de diversas outras unidades integradas ao tecido urbano central e que abrigam seu caráter de infraestrutura social, implicando na comunicação direta com a população da cidade do Rio de Janeiro. Na UFSC (B), os projetos de extensão comunitária estão concentrados majoritariamente em seu campus sede, integrado à malha viária do Bairro Trindade, enquanto as infraestruturas que se distribuem em outras unidades abrigam atividades de ensino e pesquisa. Em um recorte mais aproximado aos entornos da Cidade Universitária da UFRJ (C) e do Campus Trindade na UFSC (D), a análise focou na dimensão que Hajrasouliha (2017) chamou de conectividade. Utilizando a rede viária acessível a pedestres do OSM, os diagramas evidenciam a conectividade a partir dos acessos principais de cada um dos campi, em trajetos de até 30 minutos de caminhada. 

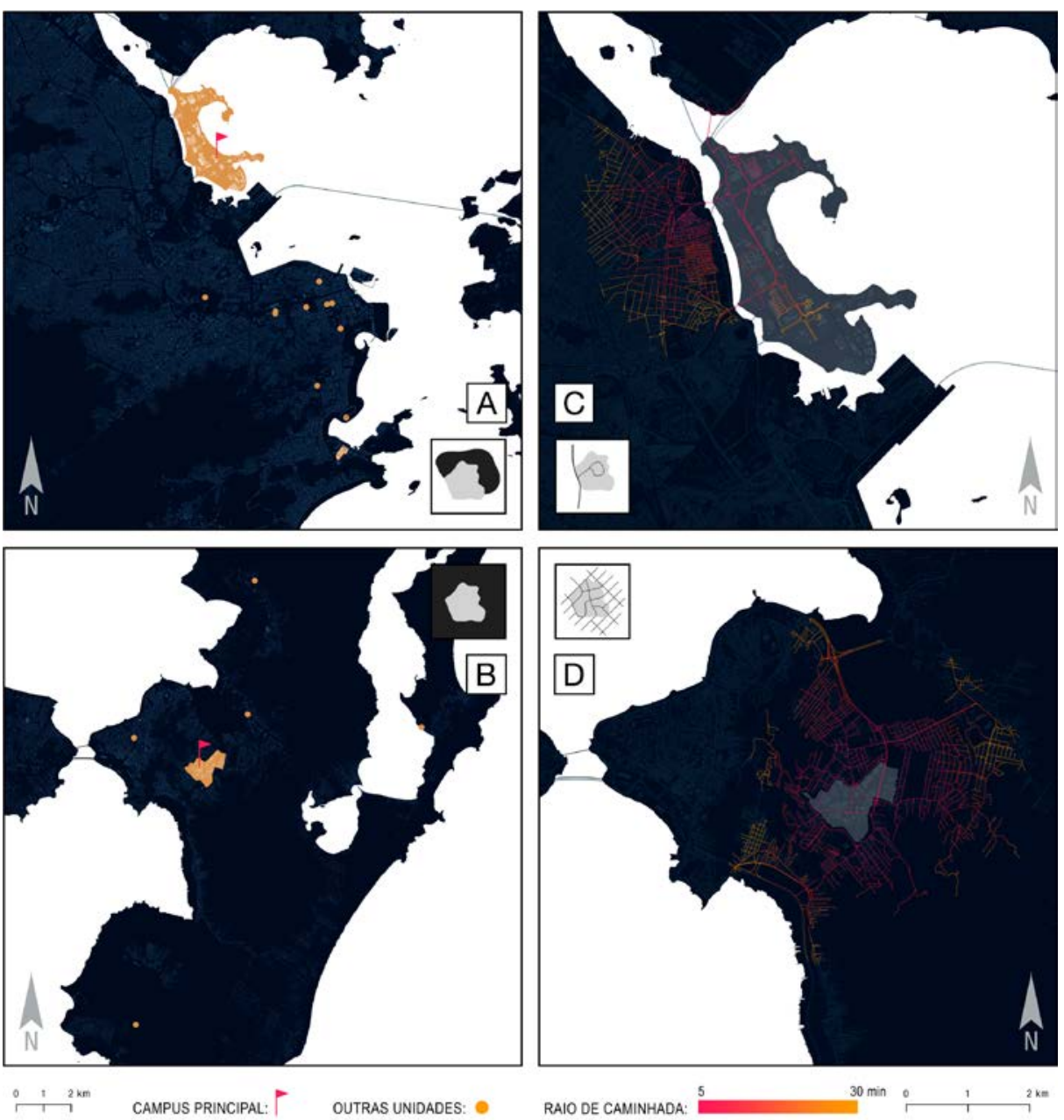

Figura 1. À esquerda, a localização e a dimensão contexto da UFRJ (A) e da UFSC (B). À direita, a malha viária e a dimensão conectividade da UFRJ (C) e da UFSC (D). Fonte: Autores, 2021, baseado em Hajrasouliha, 2017.

\subsection{Estudo 2}

A segunda etapa consistiu na avaliação qualitativa das infraestruturas sociais e suas derivações através da curadoria das plataformas online da UFRJ e da UFSC, avaliando ensino, pesquisa e projetos de extensão, além de seus planos institucionais. Essa avaliação considerou a presença da comunidade externa à universidade como fator determinante, sendo dispostas apenas as ações onde era ativa a participação social. A pesquisa teve como guia encontrar respostas às relações entre as ações institucionais e quais espaços 


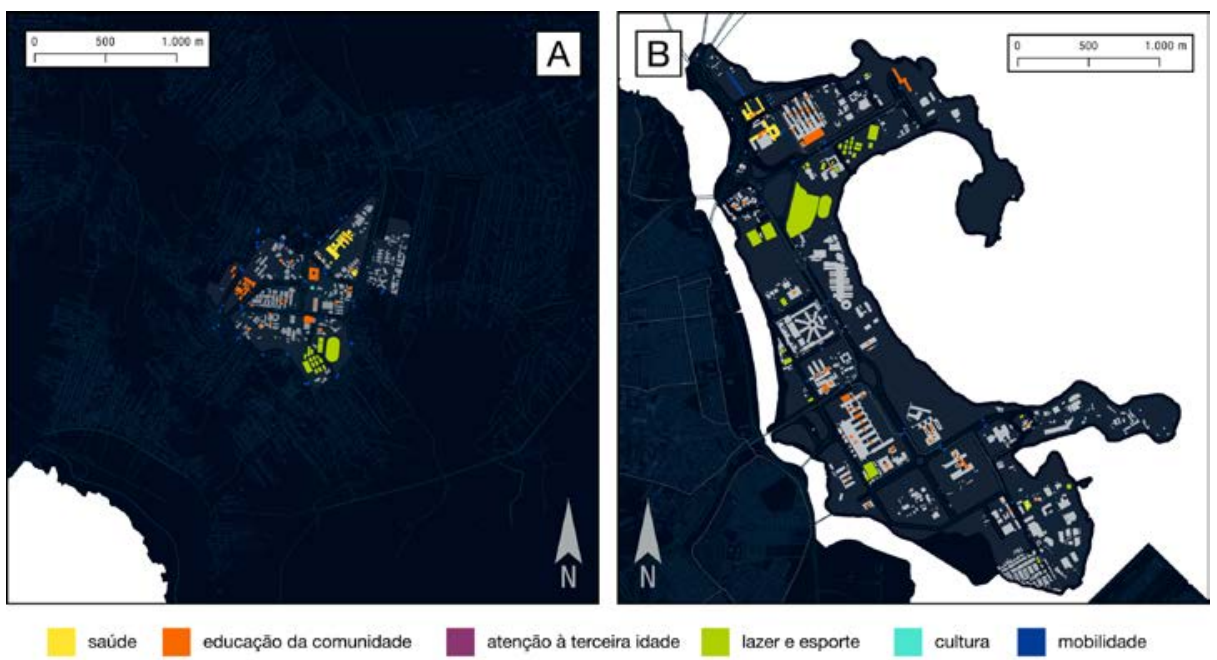

Figura 2. Localização das infraestruturas sociais da sede da UFSC (A) e da UFRJ (B). Fonte: Autores, 2021.

acolhiam suas atividades, demarcando no território universitário principal suas infraestruturas sociais (Figura 2).

Amparados pela ampla revisão de estado da arte desenvolvida por Latham e Layton (2019), as infraestruturas sociais distinguidas nas universidades foram filtradas em áreas temáticas, coluna Tipologias (Figura 3). Em seguida, consideramos os lugares que abrigam essas categorias, adotando uma separação tipológica que resultou na identificação de 24 tipologias principais, listadas na coluna Ações (Figura 3).

Para as derivações das infraestruturas sociais dentro das tipologias, foram consultadas as Cartas de Serviço de ambas as universidades (UFRJ, 2021a; UFSC, 2021a), documento que fornece aos cidadãos quais os serviços prestados pelas organizações públicas e como se dá o acesso a esses serviços. Também se coletou manualmente nas páginas dessas instituições notícias e notas relacionadas a outros programas de assistência e extensão, como na Associação Amigos do Hospital Universitário, na UFSC, ou a interação com programas da sociedade civil organizada e municípios, como no Movimento União Rio e Hospital Universitário da UFRJ (HUCFF, 2015; HUCFF, 2021; HU-UFSC, 2018; UFRJ, 2021b; UFRJ, 2021c; UFSC, 2021b). Dessa filtragem, foram obtidas ações vinculadas às tipologias levantadas. Novamente, no caso da tipologia hospital universitário, foram levantadas cinco ações principais: atendimento ambulatorial; atendimento emergencial; 

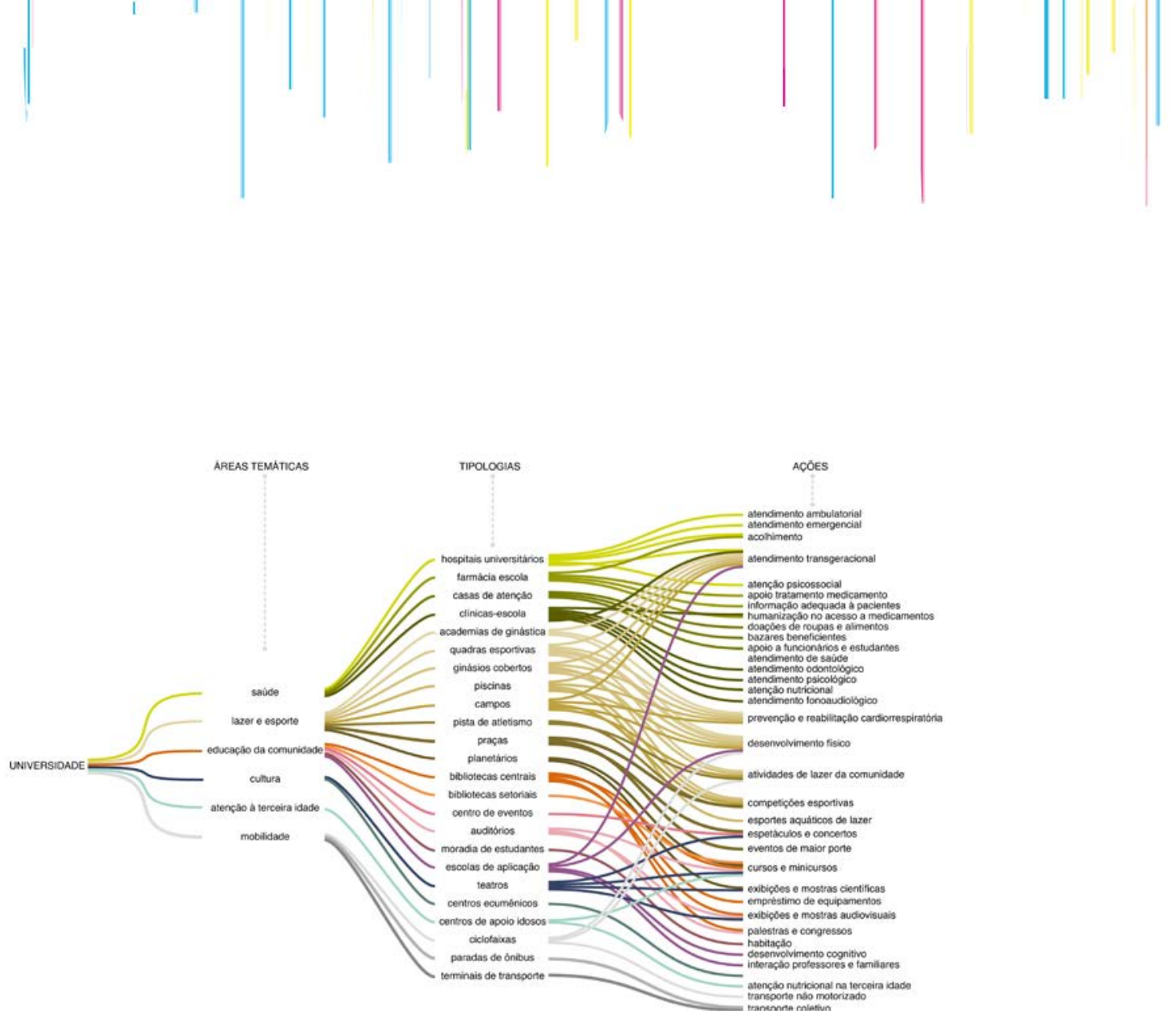

Figura 3. Diagrama de Sankey indicando as áreas temáticas, as tipologias e as ações identificadas nos sistemas das universidades. Fonte: Autores, 2021.

acolhimento humanizado; atendimento transgeracional; atenção psicossocial. O mesmo procedimento foi realizado para cada uma das tipologias.

\subsection{Estudo 3}

Considera-se aqui uma visualização espacial das informações previamente identificadas, aplicado ao Campus Trindade como protótipo pela característica de concentrar maior parte das dinâmicas da UFSC. Enquanto as tipologias foram agrupadas e georreferenciados no QGIS, as ações foram organizadas no Kepler.gl em circuitos tecendo o sistema de infraestruturas sociais do campus.

As conexões entre as tipologias formam uma rede pela interpolação dos circuitos com mais sobreposições de ações, através da Triangulação de Delaunay. Deste modo, na Figura 4 estão: A) atendimento transgeracional; B) desenvolvimento físico; C) cursos e minicursos; D) competições esportivas; E) atividades de lazer da comunidade; $F$ ) prevenção e reabilitação cardiorrespiratória; G) exibições e mostras audiovisuais; H) e espetáculos e consertos. Em I), todos os circuitos anteriores são sobrepostos. 

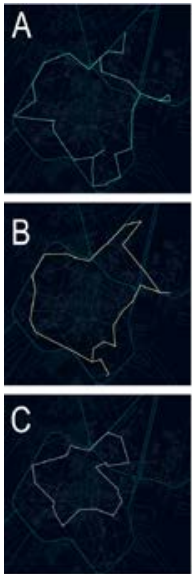

D

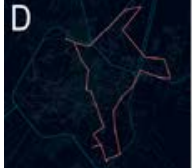

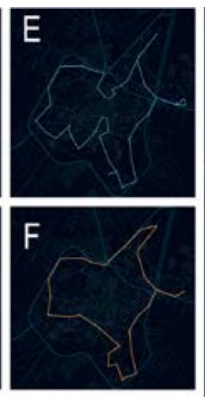

G

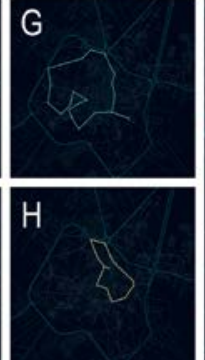

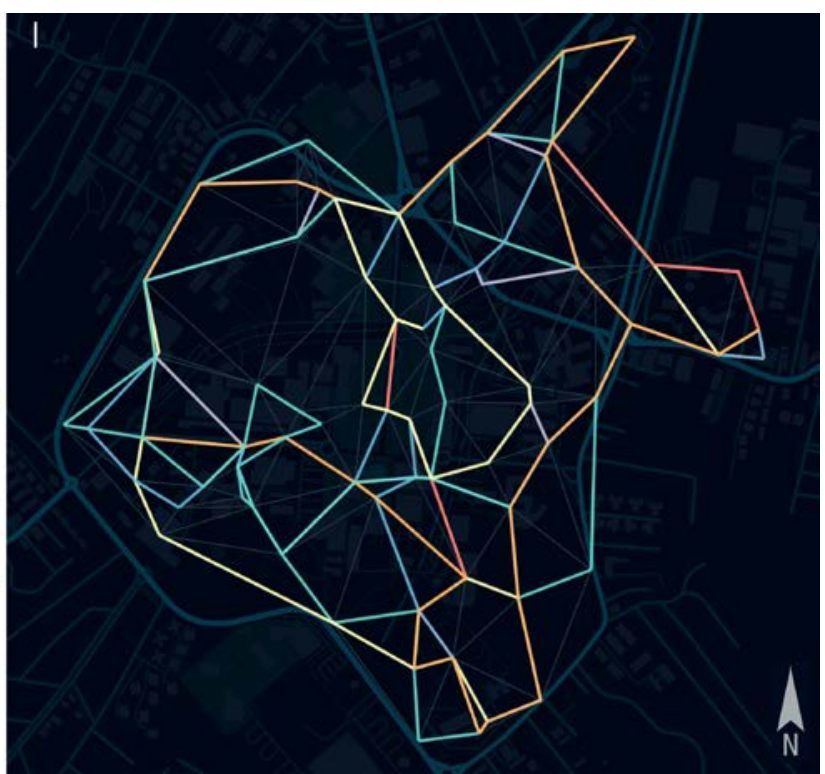

4

Figura 4. Infraestruturas sociais conectas através das sobreposições em diferentes tipologias no campus universitário. Fonte: Autores, 2021.

\section{Discussão}

Os resultados demonstram como o papel de coesão social das universidades públicas é amplo e relacional, visto que, além da provisão educacional dos estudantes, atuam na interação comunitária com a cidade. Enquanto os diagramas espaciais põem em evidência a conectividade entre o desenho do campus e o entorno, os diagramas organizacionais representam a capilaridade das infraestruturas sociais nas interseções existentes entre as ações promovidas pelas universidades.

No Estudo 1, resguardadas as similaridades institucionais, ressalta-se a diferença considerável de configuração urbana dos dois campi que, tanto pelo aspecto contextual como conectividade (Figura 1), influencia diretamente no alcance das infraestruturas acadêmicas na sociedade. Em um sentido qualitativo, nota-se que a UFRJ tem uma característica mais expansiva em relação à cidade, ao passo que a UFSC acaba sendo mais contida em seu campus sede. As imagens reforçam as diferentes expressões sociais que a configuração urbana do campus pode estabelecer com a comunidade. A localização geográfica e edilícia da universidade - isolada ou distribuída - e a trama de caminhos que conectam o campus e a cidade podem determinar o quão fortalecidas estão as correlações entre suas infraestruturas sociais. 
Estas correlações tem ainda, como estrutura basilar, os serviços oferecidos pelas universidades de modo geral, desde a atenção extracurricular dada aos estudantes até a aplicabilidade dos projetos de pesquisa. Este fato direcionou a decisão de extrapolar a análise dos Estudos 2 e 3 para além dos projetos de extensão, embora a comunicação entre essa prática acadêmica e a sociedade seja diretamente intencional e efetiva.

No Estudo 2, uma visão sistêmica das infraestruturas, focada na tipologia e que ultrapasse os três pilares que orientam as universidades (ensino, pesquisa e extensão) mostrou-se mais adaptada aos novos paradigmas científicos contemporâneos (Figura 3). Em outras abordagens, essa visão transdisciplinar pode ser também aplicada aos vínculos de sociabilidade necessários às infraestruturas sociais e que permeiam todo um sistema de áreas livres que extrapola o contexto urbano.

A conectividade acadêmica e social fica mais clara no Estudo 3. A setorização é superada por uma rede de eixos distribuídos pelo campus (Figura 4). Ao concentrar a maioria dos serviços oferecidos à comunidade no Campus Trindade, por exemplo, a UFSC oferece uma distribuição equilibrada dos aspectos de sociabilidade no seu espaço físico. A sobreposição dos circuitos de ações ilustrados realça que o território universitário tem um papel mais complexo do que as suas segmentações institucionais podem deixar transparecer, em uma atuação sistêmica entre acadêmicos e sociedade civil.

\section{Considerações Finais}

Pela ênfase gráfica e discussão sistemática, o caráter híbrido deste trabalho projeta uma percepção efetiva das infraestruturas sociais distribuídas nos territórios universitários. Expandindo o campo das relações, os diagramas buscaram estender esta percepção perpassando sistemas sociotécnicos entrelaçados em redes de componentes físicos, sociais, e institucionais. Observa-se, assim, como a capilaridade dessas infraestruturas nas cidades permite que elas componham partes decisivas dos sistemas complexos urbanos. Tal entendimento ampara-se ainda na complexidade que as universidades públicas podem tecer vinculando atores sociais a seus ambientes e que, como afirmado em Lansing (2003), podem parecer muito diferentes dos assuntos costumeiramente estudados pelos cientistas sociais. Conforme Latham e Layton (2019), a defesa desses espaços inclui a documentação do funcionamento das infraestruturas sociais e como podem ser vivenciadas democraticamente.

A disposição sistêmica das infraestruturas reforça a presença da universidade enquanto entidade urbana, servindo como importante suporte 


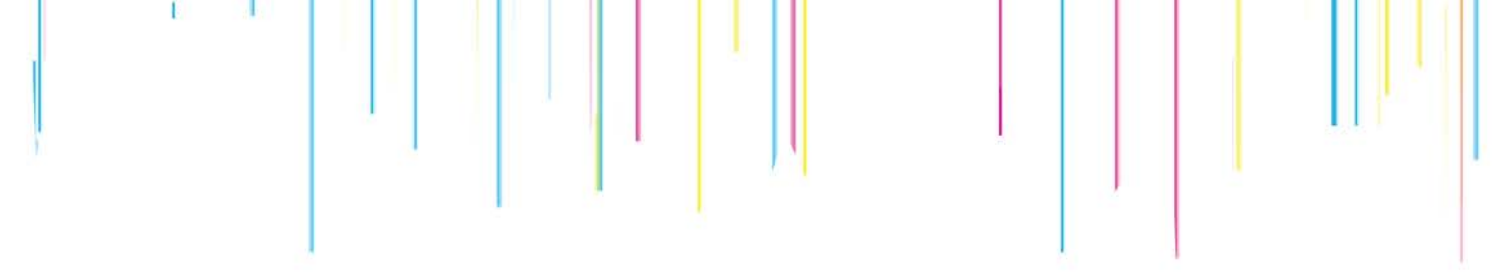

social às municipalidades. A temática ganha o aspecto transdisciplinar, intencionado como postura para o trabalho, quando a visualização das correlações passa a ser determinante para a resiliência dessas infraestruturas. Para Loukissas (2019), todos os dados são inevitavelmente amarrados aos locais, instituições, processos e pessoas que moldam sua significância e uso. Ele argumenta que, além do simples acesso, a compreensão desses registros requer a localidade dos dados e o entendimento em seus vínculos de origem, considerando as diferentes maneiras pelas quais as instituições valorizam seus sistemas informacionais.

Essa lógica deve ser amplamente aplicada na sociabilidade nas infraestruturas das universidades após a crise sanitária da COVID-19, restabelecendo os elos vitais que conectam a comunidade acadêmica, a sociedade e os sistemas ambientais. Na qualidade de concentradores sociais e transmissores de conhecimento, o papel dialético dos espaços acadêmicos amplia o caráter pedagógico e transformador materializado nos espaços de aprendizagem (Cleveland, 2011). A compreensão de como suas ações se corelacionam e estão intrincadas em seu espaço edificado oferece apontamentos mais precisos, social e territorialmente, acerca da importância do campus enquanto infraestrutura social. A visualização destas dinâmicas permite 0 reconhecimento de padrões até então pouco visíveis no território universitário. Conferiu-se aos diagramas, portanto, a tarefa de iluminar as interações sociais em direção a um campus mais plural, inclusivo e conectado, indispensável ao complexo sistema da sociedade.

Agradecimentos. Conselho Nacional de Desenvolvimento Científico e Tecnológico (CNPq - Proc. $\left.\mathrm{n}^{\circ}: 424268 / 2018-3\right)$ e Coordenação de Aperfeiçoamento de Pessoal de Nível Superior (CAPES - Código de Financiamento 001).

\section{Referências}

Alberto, K. C. (2003). Três projetos para uma Universidade do Brasil [Dissertação de Mestrado, Universidade Federal do Rio De Janeiro].

Biblioteca Universitária Universidade Federal de Santa Catarina. (2021). Serviços. https://portal.bu.ufsc.br/servicos/

Cleveland, B. W. (2011). Engaging spaces: Innovative learning environments, pedagogies and student engagement in the middle years of school [Tese de Doutorado, University of Melbourne]. http://hdl.handle.net/11343/36515

Hajrasouliha, A. H. (2017). Master-planning the American campus: Goals, actions, and design strategies. Urban Design International, 22(4), 363-381. https://doi.org/10.1057/s41289-017-0044-x 
Hospital Universitário Clementino Fraga Filho. (2015). Carta de serviços ao cidadão. http://www.hucff.ufrj.br/assistencia/9-sem-categoria/865-carta-de-servicos-ao-cidadao

Hospital Universitário Clementino Fraga Filho. (2021). Voto de gratidão: Movimento União Rio. http://www.hucff.ufrj.br/noticias/destaque/1627-voto-de-gratidao

Hospital Universitário Polydoro Ernani de São Thiago. (2018) Carta de serviços ao cidadão.http://www.hu.ufsc.br/setores/wpcontent/uploads/2018/05/CARTA_AO_ CIDADAO_2018.pdf

Klinenberg, E. (2018). Palaces for the people: How social infrastructure can help fight inequality, polarization, and the decline of civic life. Crown.

Kos, J. R., Pavan, L. H., \& Mangrich, C. P. (2020). Potencial cívico do Campus: A Universidade Federal de Santa Catarina e a democracia na cidade. Arquitecturas del Sur, 38(58), 80-97. https://doi.org/10.22320/07196466.2020.38.058.05

Lansing, J. S. (2003). Complex adaptive systems. Annual review of anthropology, 32(1), 183-204. https://doi.org/10.1146/annurev.anthro.32.061002.093440

Latham, A., \& Layton, J. (2019). Social infrastructure and the public life of cities: Studying urban sociality and public spaces. Geography Compass, 13(7), e12444. https://doi.org/10.1111/gec3.12444

Loukissas, Y. A. (2019). All data are local: Thinking critically in a data-driven society. MIT Press.

Neckel, R., \& Küchler, A. (2010). UFSC 50 anos: Trajetórias e desafios. Editora UFSC.

Pinto, G., \& Buffa, E. (2009). Arquitetura e educação: câmpus universitários brasileiros. EdUFSCar.

Taylor, I. (Ed.). (2016). Future Campus. Routledge.

Universidade Federal de Santa Catarina. (2021a). Carta de serviços ao cidadão. https://cartadeservicos.paginas.ufsc.br/files/2021/04/Carta_de_Serviços_2021.pdf

Universidade Federal de Santa Catarina. (2021b). Consulta de projetos. https://sigpex.sistemas.ufsc.br/publico/consultaSemSigilo.xhtml

Universidade Federal do Rio de Janeiro. (2021a). Carta de serviços. https://cartadeservicos.ufrj.br

Universidade Federal do Rio de Janeiro. (2021b). Sistema de bibliotecas: Todas as bibliotecas. https://www.sibi.ufrj.br/index.php/bibliotecas/todas-as-bibliotecas

Universidade Federal do Rio de Janeiro. (2021c). Visualização Pública de Ações de Extensão. https://portal.ufrj.br/Inscricao/extensao/acaoExtensao/filtro

Winner, L. (1980). Do Artifacts Have Politics? Daedalus,109(1), 121-136. 